\title{
An Energy Efficient Message Control Scheme for Wireless Sensor Actuator Networks
}

\author{
Seungcheon $\mathrm{Kim}^{1}$ and Jinhong $\mathrm{Kim}^{2}$ \\ ${ }^{1}$ Dept. of Information \& Communication Engineering, \\ Hansung University, Seoul, Korea \\ ${ }^{2}$ Department of Computer Engineering, Hansung University \\ \{kimsc,jinhkim\}@hansung.ac.kr
}

\begin{abstract}
Wireless sensor networks has been evolving toward wireless sensor actuator networks (WSAN), which provide more dynamic services based on events from sensor nodes. Actuators in WSANs are very energy sensitive device since they usually consume more energy than sensor nodes. And usually WSANs are based on the event reliability to support more accurate services. The optimum number of event notification messages from sensor nodes is very crucial part for ideal/advanced service reliability in WSANs. In this paper, we analyze how the number of event notification affects the energy consumption of actuator node in WSAN. Furthermore, a scheme that maintains WSAN staying in a low energy consumption state is proposed.
\end{abstract}

Keywords: WSAN, event reliability, service reliability, energy consumption, actuator

\section{Introduction}

Sensor network usually refers to the network that provides information services by using sensing information from sensor nodes [1]. To provide sensing information, it should have an infrastructure of sensor nodes and a sink node, which deals with delivery and processing of the sensing information. These efforts have resulted in the creation of Zigbee, IEEE 802.15.4, as PHY and MAC of wireless sensor networks (WSN). In addition, lots of works are concentrated in providing Internet services in WSNs with IPv6 [2]. But now, WSN is evolving toward the wireless sensor actuator network (WSAN) that can react promptly to the event-ofinterest, based on the sensing information from sensor nodes. In WSANs, actuator nodes are required to do specific actions or services after exchanging query or response messages with sensor nodes [3]. The reliability is significant especially in WSAN, which can be categorized into the network reliability, data transmission reliability, and the service reliability.

Service reliability means how the sink or actuator node can verify if the desired event occurs. Usually, the number of event notification messages is used to verify and confirm that the intended event happens [4].

At a later point in time, quality of service (QoS) has been an issue dependent on the services of WSNs. One of the main concerns in WSN is how to reduce the energy consumption when it exchanges sensing information. Since the energy consumption is directly connected with the life time of the sensor networks, nearly all the searches have been done from MAC to Application Layers.

When it comes to WSANs, the energy consumption in WSAN is also a crucially important matter especially in regards to/in reference to network life time. Usually, an actuator node 
consumes more energy than normal sensor nodes since it has to perform a specific action as a reaction to the events. Describe the events performed by actuator node. The more frequently the actuator node reacts to the unwanted event, therefore, the shorter the network life time becomes [5]. Therefore, we need to find out novel mechanisms to reduce the energy consumption in WSAN.

This paper is focused on investigating the proper number of event notification messages that satisfy the service reliability with minimum energy consumption of actuator node in WSAN and provides a scheme to reduce the energy consumption in WSAN.

\section{Wireless Sensor Actuator Network}

\section{A. Reliability in WSAN}

Reliability in WSN/WSAN can be categorized as Network reliability, Data transmission reliability and Service reliability.

First, the network reliability of WSAN means the network stability itself. WSN/WSAN can change its shape or configurations when its purpose of service has been changed or modified. Even though those changes happen, WSN/WSAN should be operated stably.

Second, the data transmission means to deliver the sensing data safely and reliably from sensor node to sink node. For this, we need to reconsider the way the data is transmitted in wireless sensor network environments. Typically there are three methods for data delivery: End-to-End Delivery, Hop-to-Hop Delivery and Cache Mode Delivery. Those are probably compared in aspect of reliability and efficiency. And also it should be revised to deal with the transmission errors for the data transmission reliability. Considerations on the use of ACK, NAK or Timer should be done for better transmission reliability.

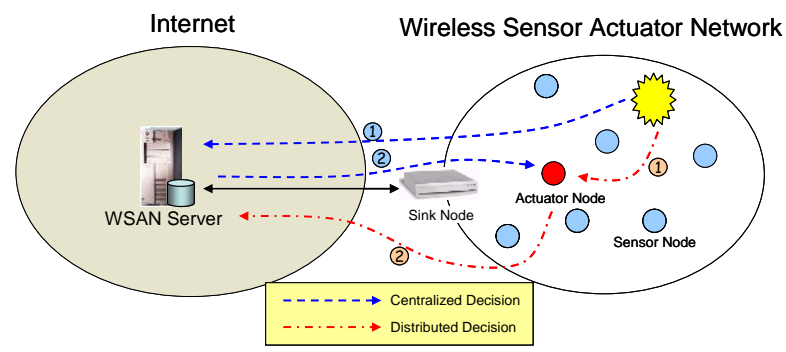

Figure 1. Decision making in WSAN

Lastly, the service reliability or event reliability is very far from the data transmission reliability in WSAN. The event is the most important interest in WSAN, where the specific reactions are related to the specific events. The service reliability, therefore, means that the actuator node can verify whether it receives proper events from sensor nodes. Usually WSANs are composed of lots of sensor nodes. The event reliability depends on the number of event notification messages and confirming messages from sensor nodes. Sometimes sensor nodes can send a notification message on a wrong event. Therefore actuator node is recommended to react when it receives a certain number of notification messages from sensor node to identify the event. 


\section{B. Sensor-Actuator Coordination}

The first thing we should think about in WSAN is how to assign an actuator node to the corresponding sensor nodes considering the reacting pattern of actuator node over the event. To determine the mutual relationship between sensor node and actuator node, we should look into how many actuator nodes are required to respond to the event notification and which actuator node are to be selected in the end. And also we need to categorize the event according to the task that is assigned to the actuator in WSANs.

\section{Single-Actuator Task vs. Multi-Actuator Task}

The most general example of WSANs is the single actuator task (SAT) that is performed by single actuator on an event. In this case, the actuator normally performs a reaction towards the event after exchanging some messages with sensor nodes about the event.

On the contrary, multi actuator task (MAT) requires actuators to do a more complicated task than SAT. In this case, task for actuators might be able to be assigned according to the mutual communication between sensor node and actuator nodes and the proper reaction plan for a specific event can be changed after communication among actuator nodes.

\section{Centralized Decision vs. Distributed Decision}

The decision making method in WSANs can be divided into centralized decision (CD) and distributed decision (DD). In CD, the event is notified to the server in the information center through sink node and the server determines how the actuator reacts to the event as shown in Figure 1.

In DD, the actuator has the right to decide whether it will perform a reaction or not when it receives the event notification from sensor nodes. The result of reaction is reported to the server through sink node by the actuator node.

In $\mathrm{DD}$, the reaction is much prompter than in $\mathrm{CD}$ but the actuator node takes the responsibility of wrong decision of the event, which result in shortening the life time of actuator and WSAN since the energy consumption of reaction is much larger than the energy consumption needed for communication.

\section{Analysis of the Event Reliability}

In WSAN, the number of notification messages for an event is definitely related to the energy consumption of actuator, which can affect the whole WSAN. Here we are going to investigate the relationship between the number of notification message and the energy consumption of actuator node.

\section{A. Probability of receiving $k$ messages during $\square \square$ in Actuator}

Assuming that every sensor node can send only one notification message when if detects an event, the probability of receiving k-messages from n-sensor nodes is possibly calculated as follows.

$$
P_{e}(k)=\left(\begin{array}{l}
n \\
k
\end{array}\right) p^{k}(1-p)^{n-k}, \text { where } p \text { is the event detection probability in sensor node. }
$$

If we assume that the average event rate is $\square$ and the interval time of each event is independent, we can use exponential distribution for the probability density function of the inter- 
val between two events in WSANs. Then, the probability of event during a specific time can be described like this.

$$
P_{\tau}=\int_{0}^{\tau} \frac{1}{\lambda} e^{-\lambda t} d t=1-e^{-\lambda \tau}
$$

Therefore, the probability that the actuator could receive $k$ messages from n-sensor nodes during $\square$ is finally described like this.

$$
P_{e}(k)=\left(\begin{array}{l}
n \\
k
\end{array}\right) p_{\tau}{ }^{k}\left(1-p_{\tau}\right)^{n-k}, \text { where } P_{\tau}=1-e^{-\lambda t}
$$

\section{B. Power consumption in Actuator}

The power consumption in actuator $\left(E_{a v}\right)$ is composed of the energy $\left(E_{a c t}\right)$ that is needed for action for specific service and the energy $\left(E_{c}\right)$ for exchanging data with sensor nodes.

$$
E_{a v}=E_{a c t}+E_{c}
$$

$E_{T}$ requires actuator node to receive more than the threshold $(T)$ number of messages from sensor nodes to verify the event in WSANs. If the number of messages in actuator node is less than $T$, actuator is supposed not to react on the event notification messages from sensor node and thus it consumes only communication energies needed to exchange only k messages with sensor nodes. Usually the energy for actuator to perform a service in WSANs is more than 10 times as big as the one needed for communication. Therefore, the average energy consumption in actuator is possibly described like this.

$$
\begin{aligned}
E_{a v} & =\operatorname{Prob}[k<T] \times E_{c}+\operatorname{Prob}[k \geq T] \times\left(E_{a v}+E_{a c t}\right) \\
& =\sum_{i=1}^{T-1} p_{e}(i) \times E_{c}+\sum_{i=T}^{n} p_{e}(i) \times\left(E_{c}+E_{a c t}\right)
\end{aligned}
$$

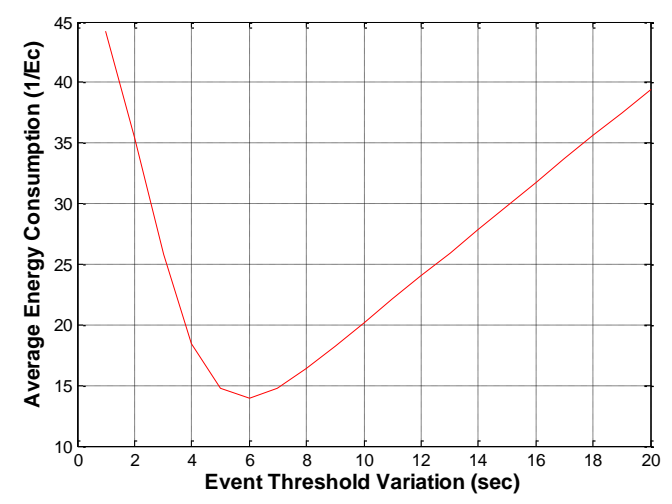

\section{Figure 2. Variation of energy consumption}

When the number of sensor nodes is set to 20 and the average arrival rate of notification message is set to $0.01 / \mathrm{sec}$ and the duration time of accepting notification messages in actuator is set to $2 \mathrm{sec}$, the result is shown in Figure 2. 


\section{Proposed Scheme}

Consequently we found that the number of the notification messages is connected to the energy consumption of the actuator in WSAN. Based on the information we found between the number of the notification messages and the energy consumption of actuator in WSAN, we propose a method that can regulate the threshold number of notification messages for the event to minimize the energy consumption of the actuator. The idea is described in Figure 3. As shown in the Figure 3, after receiving the notification messages from sensor nodes, actuator needs to start the timer for event checking and count the number of event notification message to see if it can be considered as a real event. Upon seeing the number of messages going over the threshold, it would perform the scheduled action for the corresponding event. Otherwise, it would ignore the notification messages from sensor nodes and continue to count the number of the messages and finally update the information required to calculate the threshold of notification messages for an event.

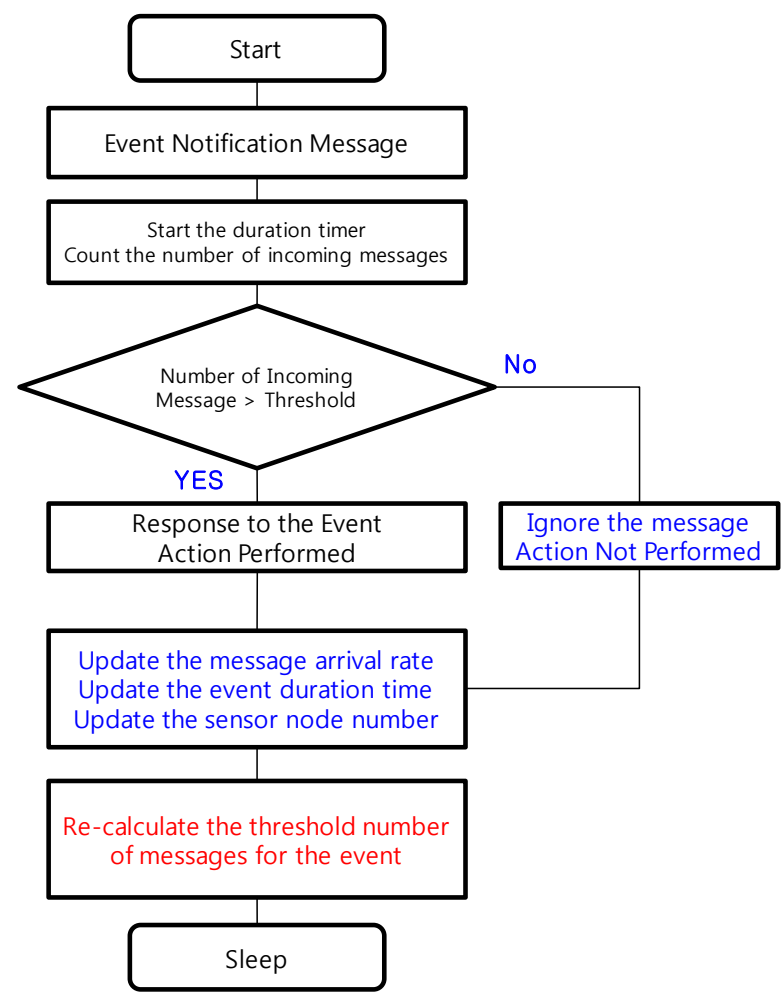

Figure 3. Proposed Algorithm

With the updated information about arrival rate of event notification message, event checking duration and the number of sensor nodes, the proper number of event notification messages for minimum energy consumption is calculated with the equation (3). This is also updated in an actuator for later detection of an event.

\section{Numerical/Simulation Results}

An experiment has been performed to see the performance of the proposed scheme. The experiment was done with MATLAB based on the equation (3). For the experiment the parameters was assumed as follows. 


\begin{tabular}{|l|l|}
\hline Energy consumption for transmitting packets $\left(E_{c}\right)$ & $1 \mathrm{~mW}$ \\
\hline Energy consumption in Actuator $\left(E_{\text {act }}\right)$ & $20 \mathrm{~mW}$ \\
\hline Event happening rate in sensor node $(\square)$ & $0.04 \sim 0.08$ \#/sec \\
\hline Sensor nodes around actuator $(N)$ & 30 \\
\hline Measuring duration $(\square)$ & $5 \mathrm{sec}$ \\
\hline
\end{tabular}

The value of threshold ' 1 ' means that the actuator reacts whenever it receives every event notification message from sensor nodes. Therefore, the energy consumption will increase as the event happening rate increase. This is shown in Figure 4.

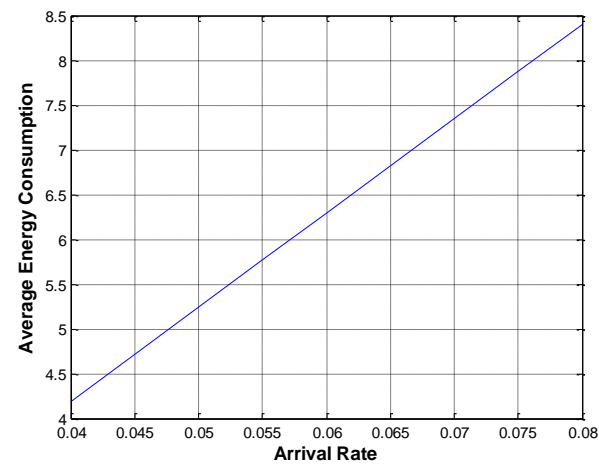

Figure 4. Energy consumption (Threshold 1)

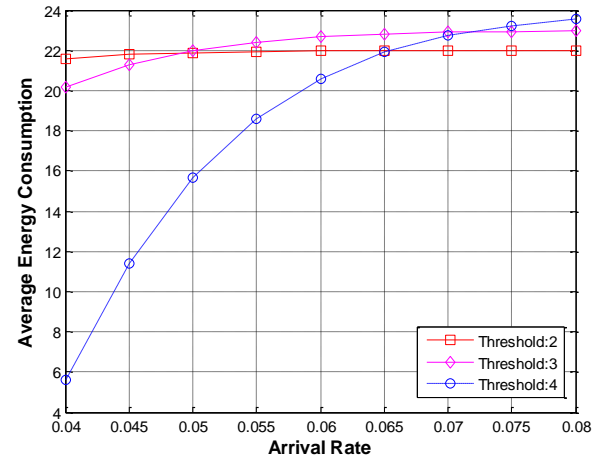

Figure 5. Average energy consumption

We have varied the value of the event happening rate to see how the energy consumption in actuator is affected by the event rate. Like in Figure 5, the energy consumption was shown different depending on the threshold value. The threshold value 4 means that the actuator reacts only when it receives 4 messages from sensor nodes during the specific time, $\square$. Therefore we were able to see the difference of energy consumption among different threshold values. But this was also affected by the event rate and is expected to be affected by the duration of $\square$. This would be the future research work with the more improvement of the proposed scheme.

\section{Conclusions}

In WSANs, the number of notification messages for an event is definitely related to the energy consumption of actuator, which can affect the life time of WSANs. In this paper, we 
investigated the relationship between the number of notification messages and the energy consumption of the actuator node. In addition, we proposed a new scheme that can regulate the threshold of event notification messages for an event and reduce the energy consumption of an actuator in WSANs. The proposed scheme is only used in an actuator and does not affect the other sensor nodes in WSANs. And also it can serve as a self-adaptive scheme for better performance. With minor improvements, the proposed scheme is expected to contribute to alleviating the burden of energy consumption problem in WSANs or WSN.

\section{Acknowledgements}

This research was financially supported by Hansung University.

\section{References}

[1] I. F. Akyildiz, W. Su, Y. Sankarasubramaniam and E. Cayirci, "A Survey on Sensor networks", IEEE Communications Magazine, (2002) August.

[2] I. F. Akyildiz and Kasimoglu, "Wireless sensor and actor networks: research challenges", Ad Hoc Networks, vol. 2, no. 4, (2004), pp. 351-367.

[3] J. Yick, B. Mukherjee and D. Ghosal, "Wireless sensor network survey", Computer Networks, vol. 52, Issue 12, (2008) August 22, pp. 2292-2330.

[4] F. Xi, "QoS Challenges and Opportunities in Wireless Sensor/Actuator Networks", Sensors, vol. 8, (2008), pp. 1099-1110.

[5] F. Xia, Y. -C. Tian, Y. Li and Y. Sun, "Wireless Sensor/Actuator Network Design for Mobile Control Applications", Sensors, vol. 7, (2007), pp. 2157-2173 
International Journal of Control and Automation

Vol.7, No.3 (2014) 